\title{
Staged Surgical Reconstruction With Skin Expansion of A Marjolin's Ulcer With Better Recovery: a Case Report
}

\section{Xudong Xie}

Wuhan Union Hospital

Liangcong $\mathrm{Hu}$

Wuhan Union Hospital

Bobin Mi

Wuhan Union Hospital

Hang Xue

Wuhan Union Hospital

Adriana C. Panayi

Wuhan Union Hospital

Yuan Xiong

Wuhan Union Hospital

\section{Lang Chen}

Wuhan Union Hospital

Chenchen Yan

Wuhan Union Hospital

Ze Lin

Wuhan Union Hospital

\section{Wu Zhou}

Wuhan Union Hospital

Guohui Liu ( $\square$ liuguohui@hust.edu.cn )

Union Hospital, Tongji Medical College, Huazhong University of Science and Technology https://orcid.org/0000-0002-2013-1396

\section{Technical note}

Keywords: Marjolin's ulcers (MUs), Surgical Reconstruction, Skin Expansion, Better Recovery, Case Report

Posted Date: September 27th, 2021

DOI: https://doi.org/10.21203/rs.3.rs-916005/v1 
License: (c) (i) This work is licensed under a Creative Commons Attribution 4.0 International License. Read Full License 


\section{Abstract}

Marjolin's ulcers (MUs) are one of the most common tumors developed from non-healing wound with chronic inflammation or injury, specially burn scars, over many years. However, the therapy recommendations for MUs are not yet definitive. Here, we presented a case of 60 -year-old man with a chronic non-healing infected wound related to MUs in the right knee. And, the wound made a better recovery after treatment with staged operation in combination with skin expansion.

\section{Background}

MUs are the tumor that was developed by some lesions in some areas of chronic inflammation or injury over many years [1]. It is mainly found in burn scars, but is also observed in other chronic wound, such as traumatic wounds, pressure sores, venous stasis ulcers and osteomyelitic fistulas. The most classic pathological type of MUs is squamous cell carcinoma (SCC), and other cancers, including basal cell carcinoma and malignant melanoma, were sometimes reported as MUs [2, 3]. Although the MUs are rare, it could lead to potentially severe consequences due to its higher aggressive, rates of local recurrence and metastasis [4].

Currently, evidence-based therapies regarding to MUs are not recommended. In most cases, it is a preferred choice for MUs to perform wide local excision with skin grafting. Sometimes more complex reconstructions with free tissue transfer were conducted when it involved bone or vital tissues. Further, limb amputation is performed in order to control and treat the disease. In our case, we performed staged operation in combination with skin expansion for the patients under the basis of preferred method. To our knowledge, the improved operation has not been reported.

\section{Case Report}

A 60-year-old man was admitted to the Orthopedics department for treatment of a deteriorating nonhealing wound in the right knee. Multiple years prior, the patient had experienced a burn of the right thigh, with evident hypopigmentation in the scar area and surrounding skin. Subsequently, the patient had incurred a traumatic open injury in the right distal thigh two years prior to admission, and the patient selfmedicated at home with no improvement. Unfortunately, treatment was so delayed that the window of therapeutic benefit was missed and the wound was greatly expanded. Thus, the patient was admitted to hospital due to an infection and limited motion in the right knee. At the time of admission, the lesion was overlying the lateral aspect of the right knee, measuring $14 \times 9 \times 4 \mathrm{~cm}$ with a small amount of bleeding and exudate on its surface, and a medial soft tissue bulge with an ulcerated surface and lateral soft tissue contraction (Fig. 1). The surrounding tissues did not show any obvious inflammatory reactions and regional lymph nodes were not palpable. Histopathology showed highly differentiated SCC but the surrounding skin was not affected. Thus, the decision was made to perform local removal of the necrotic tissue followed by skin grafting. For the first operation (Fig. 2A), performed under spinal anesthesia, adequate removal of necrotic tissue and wound debridement was performed and the wound was 
prepared for skin expansion with Kirschner wires. Concurrently, a frozen section diagnosis was performed during the surgery, which showed highly differentiated SCC but no signs of involvement of the surrounding edges and basal margin. This was consistent with the postoperative pathology results. Three days after the first operation, skin expansion was initiated at a rate of $1 \mathrm{~mm}$ per day. Given right knee joint involvement and high stretch resistance due to surrounding skin scarring, the patient experienced unbearable pain. Therefore, nine days after the first operation, we decided to perform the second operation (Fig. 2B, C) with further debridement, removal of the Kirschner wire and covering with Vacuum Sealing Draina (VSD). A third operation was performed 16 days after the first operation (Fig. 2D), during which the patient received an autologous full-thickness skin graft after debridement. The wound was covered with a VSD. Finally, the VSD was removed at the bedside, and the transplanted skin showed good survival 10 days after the third operation (Fig. 3A). Furthermore, follow-up confirmed that the transplanted skin had complete survival 42 days after the third operation (Fig. 3B). At the two years follow-up there was excellent structural and functional recovery with adequate right knee movement, including flexionextension and walking. No evidence of recurrence was noted (Fig. 3C).

\section{Discussion}

MUs are more aggressive cutaneous neoplasms compared with other kind of SCCs. The most common risk factor was old burn scars, followed by traumatic wounds, venous stasis ulcers, pressure sores, osteomyelitic fistulas, and other chronic wounds. It is reported that approximately $2 \%$ of MUs develop from burn scars [5], and the latency period for the development of malignancy can vary from 11 to 75 years, with an average latency of 31 years [6,7]. Interestingly, the incidence of MUs in males is approximately three times higher than that in females, with the peak occurrence is in the fifth decade of life [3].

Occurrence of MU may be multifactorial, including a depressed immune system, chronic irritation and impaired immunologic reactivity to tumor cells, all of which work together to promote malignant transformation of the ulcer. Thus, as soon as MUs was suspected, biopsy of multiple areas should be considered in cases of chronic or non-healing ulcers [8]. Biopsy histopathology of MU results in identification of SCC in the majority of cases (76\%), followed by basal cell (approximately $12 \%$ ) and melanoma (approximately 6\%).

Treatment and prognosis of MU depends on tumor tissue type and whether there is co-existent metastasis. For example, treatment of well-differentiated lesions involves local excision which has a good prognosis [9]. However, patients with lymph node metastasis are better suited for wide excision or amputation, and even the ulcer might recur, especially within 3 years, resulting in a significant reduction of 3-year survival rate from 35-50\% [10]. In our case, given the patient without lymph node metastasis, the patient received the local excision of lesion. Differently, we have adopted the regimen of staged surgical reconstruction for patient to adequately remove necrotic tissue and infectious lesions, and then also tried a strategy of skin stretching before skin grafting to reduce skin transplantation area and further boost skin transplant survival. However, given the involvement of the right knee joint and surrounding 
skin scarring caused by the prior burn, simple skin expansion failed to achieve adequate results, but the wound obtained good recovery. Therefore, treatment of similar lesions located in non-joint regions may also benefit from skin stretching to promote wound repair after surgical excision.

Taken together, MUs are the rare tumor with higher aggressive, rates of local recurrence and metastasis, and wide local excision with skin grafting is the most common surgical procedure. In this case, we provided an improved treatment protocol for the MUs patient with chronic non-healing wounds, which might be beneficial to these patients.

\section{Declarations}

\section{Ethical approval and Consent}

The Wuhan Union Hospital exempts case reports from ethical approval. Written informed consent was obtained from the patient for publication of this case report and accompanying images. A copy of the written consent is available for review by the Editor-in-Chief of this journal.

\section{Data Availability Statement}

The original material in the study could be directed to the corresponding authors.

\section{Conflicts of interest}

All authors declare that they have no competing financial interests.

\section{Author contributions}

Xudong Xie, Liangcong Hu, Bobin Mi, and Hang Xue conceived and designed the research and collected these data. Xudong Xie, Liangcong Hu and Bobin Mi drafted the manuscript. Adriana C. Panayi, Wu Zhou, and Guohui Liu critically reviewed the manuscript with relevant intellectual contributions. All authors read and approved the final version of the manuscript.

\section{References}

1. Kerr-Valentic MA, Samimi K, Rohlen BH, Agarwal JP, Rockwell WB. Marjolin's ulcer: modern analysis of an ancient problem. Plastic and reconstructive surgery. 2009 Jan;123(1):184-191. https://doi.org/10.1097/PRS.0b013e3181904d86.

2. Yu N, Long X, Lujan-Hernandez JR, Hassan KZ, Bai M, Wang Y, Wang X, Zhao R. Marjolin's ulcer: a preventable malignancy arising from scars. World journal of surgical oncology. 2013 Dec 17;11:313. https://doi.org/10.1186/1477-7819-11-313.

3. Pekarek B, Buck S, Osher L. A Comprehensive Review on Marjolin's Ulcers: Diagnosis and Treatment. The journal of the American College of Certified Wound Specialists. 2011 Sep;3(3):60-4. https://doi.org/10.1016/j.jcws.2012.04.001. 
4. Alam M, Ratner D. Cutaneous squamous-cell carcinoma. The New England journal of medicine. 2001 Mar 29;344(13):975-83. https://doi.org/10.1056/nejm200103293441306.

5. Aydoğdu E, Yildirim S, Aköz T. Is surgery an effective and adequate treatment in advanced Marjolin's ulcer? Burns : journal of the International Society for Burn Injuries. 2005 Jun;31(4):421-31. https://doi.org/10.1016/j.burns.2005.02.008.

6. Copcu E, Aktas A, Sişman N, Oztan Y. Thirty-one cases of Marjolin's ulcer. Clinical and experimental dermatology. 2003 Mar;28(2):138-41. https://doi.org/10.1046/j.1365-2230.2003.01210.x.

7. Kowal-Vern A, Criswell BK. Burn scar neoplasms: a literature review and statistical analysis. Burns : journal of the International Society for Burn Injuries. 2005 Jun;31(4):403-13. https://doi.org/10.1016/j.burns.2005.02.015.

8. Asuquo M, Ugare G, Ebughe G, Jibril P. Marjolin's ulcer: the importance of surgical management of chronic cutaneous ulcers. International journal of dermatology. 2007 Oct;46 Suppl 2:29-32. https://doi.org/10.1111/j.1365-4632.2007.03382.x.

9. Hill BB, Sloan DA, Lee EY, McGrath PC, Kenady DE. Marjolin's ulcer of the foot caused by nonburn trauma. Southern medical journal. 1996 Jul;89(7):707-10. https://doi.org/10.1097/00007611$199607000-00011$.

10. Bauer T, David T, Rimareix F, Lortat-Jacob A. Marjolin's ulcer in chronic osteomyelitis: seven cases and a review of the literature. Revue de chirurgie orthopedique et reparatrice de l'appareil moteur. 2007 Feb;93(1):63-71. https://doi.org/10.1016/s0035-1040(07)90205-6.

\section{Figures}




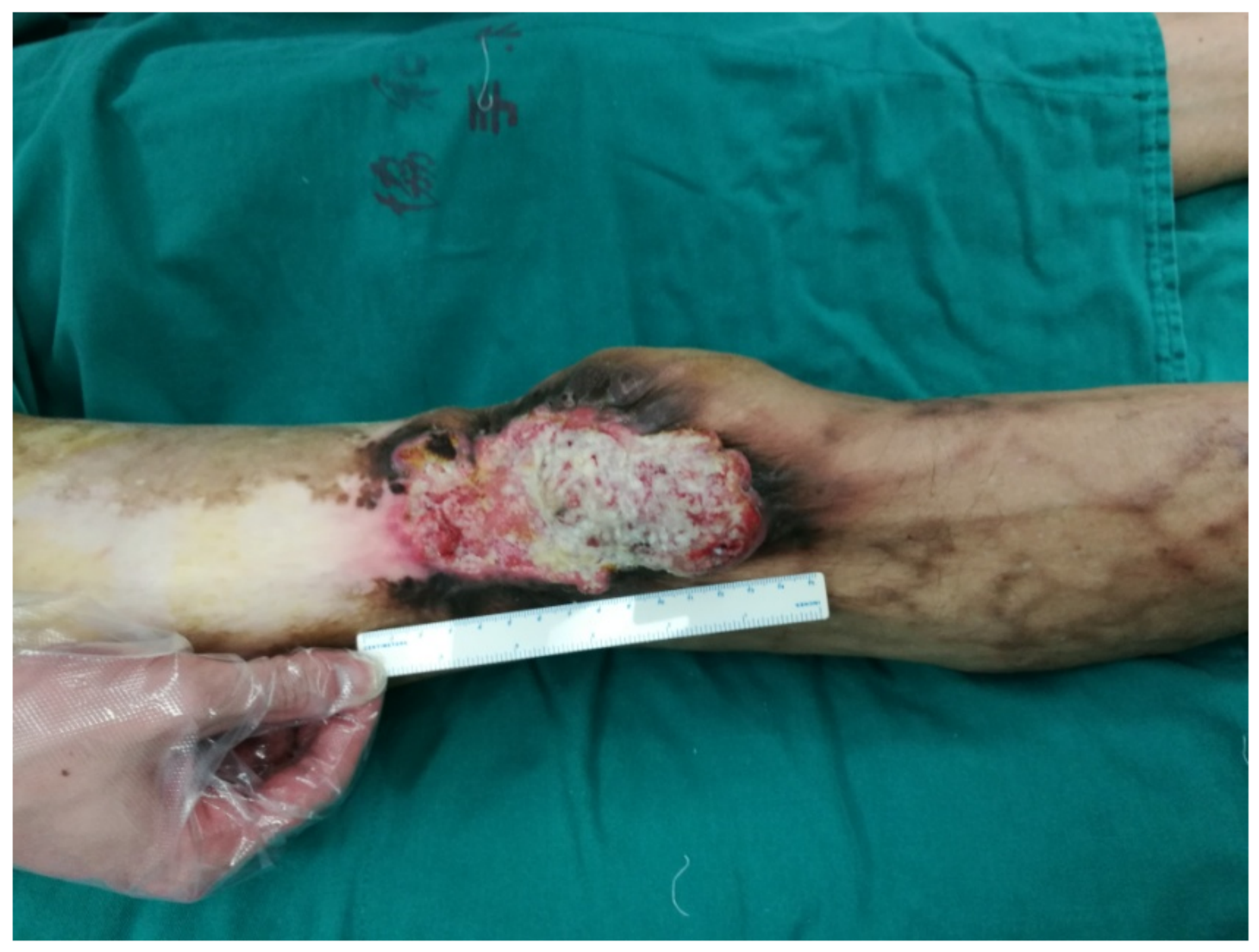

\section{Figure 1}

Initial wound appearance. A lesion measuring $14 \times 9 \times 4 \mathrm{~cm}$ with irregular borders was evident on the lateral aspect of the right knee. A small amount of bleeding and exudate on its surface was noted, with medial soft tissue bulging, an ulcerated surface and lateral soft tissue contraction. In addition, the skin surrounding the lesion had residual pigment and scarring, and the skin surface was uneven. 

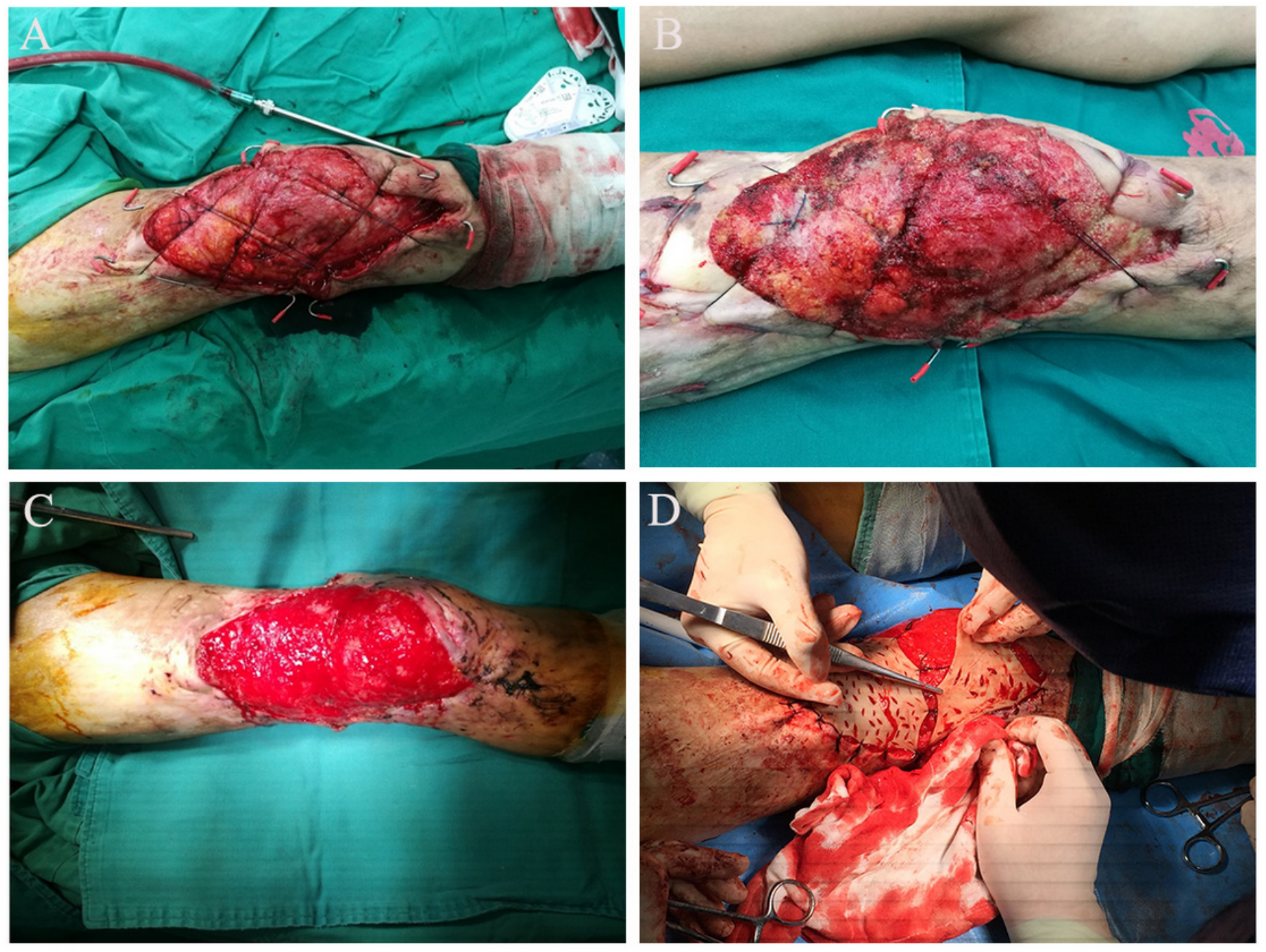

Figure 2

The process of surgical operation. (A)Wound appearance after the first operation. The patient underwent a local excision with resection margins of $\leq 10 \mathrm{~mm}$ of the surrounding tissue, including the subcutaneous fat and superficial fascia layer. Two Kirschner wires were inserted to prepare the skin for expansion. Finally, the wound was covered with vaseline gauze and dressed with a cotton pad. (B) Wound appearance before the second operation (9 days after the first operation). Partial granulation tissue formation could be seen in some areas of the wound. No shrinkage was noted in the original wound size. (C) Wound appearance after the second operation (9 days after the first operation). The two Kirschner wires were removed. The wound was debrided and covered with a Vacuum Sealing Drainage (VSD) connected to negative pressure suction. (D) During the third operation. Tissue was taken from the anteromedial skin of the contralateral thigh, and defatted to form a full-thickness graft. Multiple perforations in the skin ensured adequate tension. 

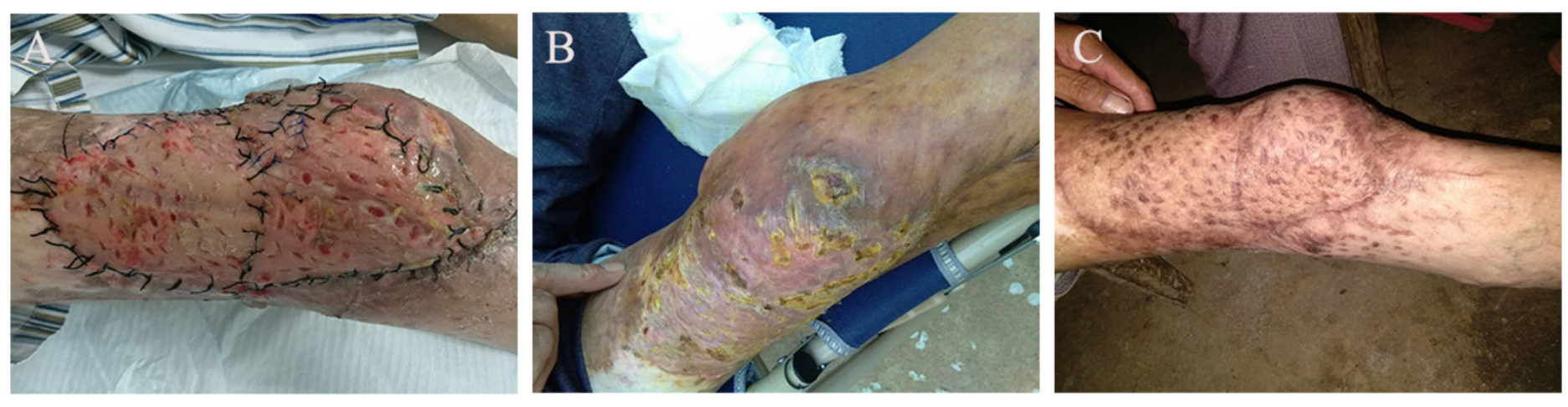

\section{Figure 3}

The wound healing after performing procedure. (A)Recipient site appearance 10 days after the third operation. The grafted skin showed good survival. A dry wound was noted with no signs of exudate or infection. (B) Recipient site appearance 42 days after the third operation. The wound had healed successfully, with evidence of local hyperpigmentation and dry scabbing. (C) Recipient site appearance two years after surgery. The wound had completely healed with no evidence of recurrence. Notable scarring and hyperpigmentation were apparent on the recipient and surrounding skin. 